\section{(2) OPEN ACCESS}

\title{
Continuous electrocardiography for detecting atrial fibrillation beyond 1 year after stroke in primary care
}

\author{
Louise Feldborg Lyckhage (i) , ${ }^{1}$ Morten Lock Hansen, ${ }^{2,3}$ Jens Christian Toft, ${ }^{4}$ \\ Susanne Lis Larsen, ${ }^{5}$ Bente Brendorp, ${ }^{6}$ Ari Mohammad Ali, ${ }^{1}$ Troels Wienecke ${ }^{1,7}$
}

- Additional material is published online only. To view please visit the journal online (http://dx.doi.org/10.1136/ heartjnl-2020-316904).

'Department of Neurology, Zealand University Hospital, Roskilde, Denmark

${ }^{2}$ Department of Cardiology, Copenhagen University Hospital Herlev and Gentofte, Hellerup, Denmark

${ }^{3}$ The Cardiovascular Research Centre, Copenhagen University Hospital, Gentofte, Denmark ${ }^{4}$ Department of Cardiology and Endocrinology, Slagelse Hospital, Slagelse, Denmark ${ }^{5}$ Borup and Viby General Practice, General practice in Region Zealand, Borup, Denmark

${ }^{6}$ Department of Cardiology, North Zealand Hospital, Hilleroed, Denmark

${ }^{7}$ Department of Clinical Medicine, University of Copenhagen, Copenhagen, Denmark

\section{Correspondence to}

Dr Louise Feldborg Lyckhage and Dr Louise Feldborg Lyckhage, Neurology, Zealand University Hospital Roskilde, 4000 Roskilde, Denmark; Ilyckhage@hotmail.com. llyckhage@hotmail.com

Received 15 March 2020 Revised 16 May 2020 Accepted 22 May 2020 Published Online First 3 July 2020

Check for updates

(C) Author(s) (or their employer(s)) 2021. Re-use permitted under CC BY-NC. No commercial re-use. See rights and permissions. Published by BMJ.

To cite: Lyckhage LF, Hansen ML, Toft JC, et al. Heart 2021;107:635-641.

\section{ABSTRACT}

Background and purpose The diagnostic benefit of using continuous ECG (cECG) for poststroke atrial fibrillation (AF) screening in a primary care setting is unclear. We aimed to assess the diagnostic yield from screening patients who previously had a stroke with a 7-day Holter monitor.

Methods Patients older than 49 years, naive to $A F_{\text {, }}$ with an ischaemic stroke over 1 year before enrolment were included. In a primary care setting, all patients were screened for AF using pulse palpation, 12-lead ECG and 7-day Holter monitoring. Further, NT-proBNP was determined at baseline.

Results 7-day Holter monitoring uncovered AF in 17 of 366 patients ( $4.6 \%(95 \% \mathrm{Cl} 2.7$ to 7.3$)$ ). The number needed to screen was 22 patients (14-37). 12-lead ECG uncovered AF in 3 patients $(0.82 \%$ (95\% Cl 0.17 to 2.4)), and 122 patients had irregular pulse during pulse palpation (33.5\% (95\% Cl 28.7 to 38.2)). When using 7-day Holter monitoring as reference standard, the sensitivity of pulse palpation and 12-lead ECG was $47 \%$ (95\% Cl $23 \%$ to $72 \%$ ) and $18 \%$ (95\% Cl $4 \%$ to $43 \%$ ). High levels $(\geq 400 \mathrm{pg} / \mathrm{mL}$ ) of NT-proBNP versus low levels $(\leq 200 \mathrm{pg} / \mathrm{mL}$ ) were not associated with AF in the univariate analysis nor when adjusted for age (OR 2.4 ( $95 \% \mathrm{Cl} 0.5$ to 8.4 ) and $1.6(95 \% \mathrm{Cl} 0.3$ to 6.0$)$ ).

Conclusions A relevant proportion of patients with stroke more than 1 year before inclusion were diagnosed with AF through 7-day Holter monitoring. Given the low sensitivities of pulse palpation and 12-lead ECG, additional CECG may be considered during poststroke primary care follow-up.

\section{INTRODUCTION}

Ischaemic stroke is a prevalent and potentially debilitating condition, where identification and treatment of potential risk factors of recurrent stroke are crucial. ${ }^{1}$ Atrial fibrillation (AF) is one of the most important risk factors 'and is associated with a five-fold increased risk of ischemic stroke. ${ }^{2}$ This risk is reducible by converting therapy from antiplatelet to oral anticoagulant treatment (OAC). ${ }^{3}$ Effective AF detection after stroke is challenging since $\mathrm{AF}$ is often paroxysmal and asymptomatic. ${ }^{4}$ Randomised clinical trials and meta-analyses have shown that increasing the time of ECG monitoring in the early phase after ischaemic stroke uncovers progressively more $\mathrm{AF}^{5-7}$ Consequently, current recommendations include use of continuous ECG (cECG) early after ischaemic stroke. ${ }^{8}$
The long-term efforts to prevent recurrent stroke are typically handled at patients' general practice (GP) clinic. Guidelines for the elderly population include opportunistic pulse palpation followed by 12-lead ECG in case of irregular pulse. ${ }^{8}$ Nevertheless, there are currently no guidelines specific to primary care poststroke AF screening. Brachmann $e t a l^{6}$ showed that monitoring patients with cryptogenic stroke with implantable loop recorder detected $\mathrm{AF}$ in $30 \%$ after 3 years compared with $12.4 \%$ after 1 year. Thus, AF could be markedly underdiagnosed even years after stroke without the use of effective systematic screening methods. Using implantable loop recorders in all patients who had a stroke does, however, seem unrealistic.

This is the first study to investigate the role of external cECG as an AF screening tool in a primary care setting beyond the first year after ischaemic stroke. In collaboration with GP clinics, our primary aim was to determine the proportion of newly diagnosed AF by use of 7-day Holter monitoring. The secondary aims included assessment of the sensitivity of pulse palpation and 12-lead ECG, using 7-day Holter monitoring as a reference standard, and assessment of the association of high versus low levels of N-terminal Pro-Brain Natriuretic Peptide (NT-proBNP) with the risk of AF.

\section{METHODS}

\section{Design, setting and data collection}

This prospective cohort study was a collaboration between the Neurovascular Center, Zealand University Hospital (NVC) and two GP clinics in the Region of Zealand, Denmark (Borup and Havdrup). Patient recruitment began in November 2016 at Borup GP clinic and in November 2017 at Havdrup GP clinic. We initially aimed to conduct all recruitment and data collection via the primary sector. Through a protocol amendment in March 2018, we improved recruitment rate by expanding the source population to all participants previously admitted to the NVC and set up facilities for data collection at the latter. Due to changes in the hospital patient record systems, data were not available for participants with stroke beyond 10 years. Consequently, we restricted time since last stroke to 1-10 years prior to inclusion for all participants, meaning some of the participants recruited via the GP clinic in Borup were excluded from analysis. Enrolment was completed in April 2019. Trained GP nurses conducted data collection in the primary sector and 
research assistants or the primary investigator conducted data collection at the NVC.

\section{Study participants}

Participants included were AF-naive, had ischaemic stroke over 1 year before enrolment and were older than 49 at stroke onset. Participants with a systemic infection or taking antiarrhythmic drugs (class I and III, digoxin, flecainide, and non-dihydropyridine calcium-channel blockers), who had cECG within 1 year before inclusion, and who had an implanted loop recorder, cardioverter defibrillator or pacemaker were not eligible. Participants with an acute infection or surgery were included at least 1 month after remission. Participants taking OAC for other indications than AF were included.

The ischaemic stroke diagnosis, established through review of patient records, was defined as evidence of previous focal central nervous system (CNS) infarction documented by clinical findings and/or acute or chronic infarction, or a relevant perfusion defect on CT or MRI. ${ }^{9}$ Only imaging related to the last stroke diagnosis was evaluated. Where imaging lacked evidence of infarction, we required neurological findings consistent with upper motor neuron lesions, such as pathological reflexes, upper extremity pronation, central facial palsy or central ophthalmoplegia, and no probable differential diagnosis. We also included participants with silent CNS infarction, detected during evaluation without certain stroke symptoms. Silent CNS infarctions are associated with increased risk of stroke. ${ }^{10}$ The subtype classification of stroke was done in accordance with the Trial of Org 10172 in Acute Stroke Treatment. ${ }^{11}$

\section{Cardiac rhythm evaluation}

Study evaluation for AF or atrial flutter included pulse palpation for irregular pulse, 12-lead ECG and 7-day cECG monitoring. All three evaluations were performed on all participants and 12-lead ECG was performed minutes before or after application of Holter equipment. Radial artery pulse palpation for pulse irregularity was done for a minimum of $20 \mathrm{~s}$ or as long as needed. ${ }^{12}$ The sensitivity and specificity of pulse palpation were $0.92 \%$ and $0.82 \%$ in previous studies. ${ }^{13} \mathrm{~A}$ two-channel 7-day Holter monitoring was conducted using the Lifecard CF (Spacelabs Healthcare, Snoqualmie, Washington, USA). The Pathfinder SL software was used for subsequent automatic AF screening and has shown a sensitivity and specificity of over 98\% and $80 \%$, respectively. ${ }^{14}$ The algorithm for AF detection was described by Haeberlin et al. ${ }^{14}$ To confirm the diagnosis, all detected episodes were adjudicated by the primary investigator and two specialist raters. A third specialist rater determined the diagnosis in cases of inter-rater disagreement. AF was defined according to accepted convention as at least $30 \mathrm{~s}$ of absolutely irregular RR intervals and the absence of p-waves. ${ }^{8}$ Due to a regional technical error, the raw Holter ECG data are unavailable, but ECG segments of all episodes of confirmed or suspected episodes of AF are saved and stored as separate PDF files.

NT-proBNP and further covariates are described in online supplementary appendix methods and table S1, respectively.

\section{Statistical analysis}

The sample size of 400 participants was obtained for ClopperPearson CI for binomial proportion. A statistician at the department of 'Production, Research \& Innovation (PFI)' in the 'Region of Zealand' conducted the sample size calculation. The online supplementary appendix describes in detail the methods used for our power calculation.

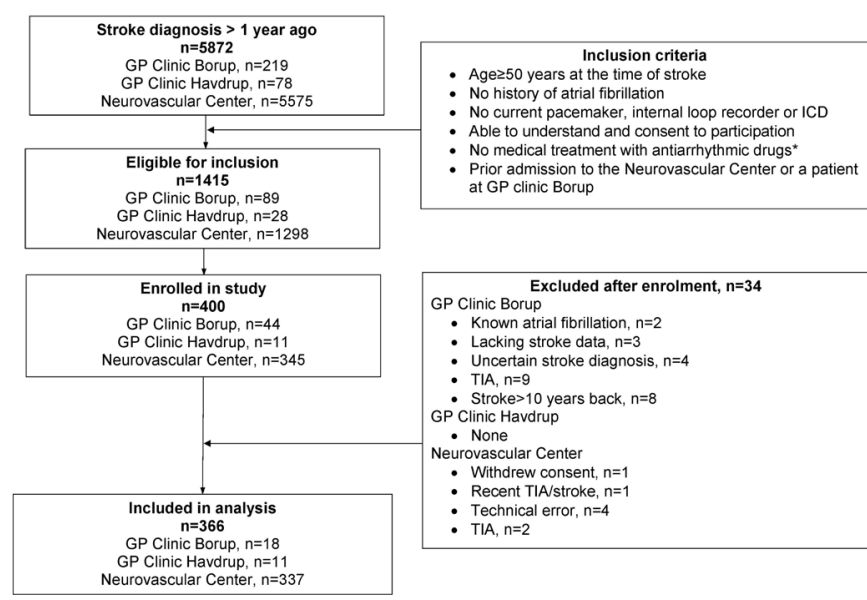

Figure 1 Flow chart of patient selection and distribution between study locations. * Class I and III, digoxin, flecainide, and nondihydropyridine calcium-channel blockers. GP clinic, general practice clinic; ICD, implantable cardioverter defibrillator; TIA, transient ischaemic attack.

Baseline statistics of categorical variables were expressed as counts and percentages. Normally and non-normally distributed continuous variables were expressed as a median with limits of IQR (Q1-Q3) and as mean and SD. The Clopper-Pearson interval method was used to determine percentages of AF (including 95\% CI) as diagnosed by 12-lead ECG, 7-day Holter monitoring and for participants with irregular pulse determined during pulse palpation. Sensitivity, specificity, and positive and negative predictive values including exact binomial confidence intervals were calculated by the epi.test function in the epiR package in RStudio. Further, the OR of AF in high versus low levels of NT-proBNP was assessed by univariable logistic regression analysis and adjusted for age. Exploratory univariable analysis for AF risk by each covariable was conducted (online supplementary appendix methods and table S2). RStudio V.1.1.453 was used for statistical analyses.

All participants gave an informed written consent.

\section{RESULTS}

\section{Study population}

Of 1424 participants eligible for inclusion, 418 (29.4\%) accepted study participation. Of these, the first 400 were enrolled. There was no significant difference in age and sex distribution between participants and non-participants (online supplementary appendix table S3). After excluding 34 participants, the study population consisted of 366 participants. Twenty-two participants were included solely based on a chronic cerebral infarct. Figure 1 illustrates the selection of participants.

\section{Baseline characteristics}

The mean age was 70 years (SD 8) and $65.6 \%$ of the participants were men. The median time from last stroke was 3.9 years (Q1Q3 2.5-5.9) and the median $\mathrm{CHA}_{2} \mathrm{DS}_{2}$-VASc score (Congestive heart failure, Hypertension, Age, Diabetes, previous Stroke/ transient ischemic attack, Vascular disease, Sex category) was 4 (Q1-Q3 4-5). No participants had mitral valve stenosis or a mechanical heart valve. Table 1 shows the baseline characteristics.

\section{AF yield}

Pulse palpation, 12-lead ECG and 7-day Holter monitoring were conducted on all participants and the median duration 
Table 1 Baseline characteristics

\begin{tabular}{|c|c|c|c|}
\hline & All, $\mathrm{N}=366$ & No atrial fibrillation, $n=349$ & Atrial fibrillation, $n=17$ \\
\hline Age, mean (SD) & $70(8.1)$ & $69.8(8)$ & $74.6(9.3)$ \\
\hline Age $\geq 75$ years & $106(29)$ & $97(27.8)$ & $9(52.9)$ \\
\hline Female & $126(34.4)$ & $124(35.5)$ & $2(11.8)$ \\
\hline Years since last stroke & $3.9(2.5-5.9)$ & $3.9(2.5-5.9)$ & $3.8(2.4-4.6)$ \\
\hline$>1$ clinical stroke or TIA & $104(28.4)$ & $101(28.9)$ & $3(17.6)$ \\
\hline $\mathrm{CHA}_{2} \mathrm{DS}_{2}$-VASc score & $4(4-5)$ & $4(4-5)$ & $5(4-6)$ \\
\hline \multicolumn{4}{|l|}{$\mathrm{CHA}_{2} \mathrm{DS}_{2}$-VASc groups } \\
\hline $2-3$ & $83(22.7)$ & $81(23.2)$ & $2(11.8)$ \\
\hline $4-5$ & $230(62.8)$ & $220(63)$ & $10(58.8)$ \\
\hline $6-7$ & $53(14.5)$ & $48(13.8)$ & $5(29.4)$ \\
\hline \multicolumn{4}{|l|}{ Comorbidities } \\
\hline Ischaemic heart disease & $27(7.4)$ & $25(7.2)$ & $2(11.8)$ \\
\hline Heart failure & $1(0.3)$ & $1(0.3)$ & 0 \\
\hline Hypertension & $255(69.7)$ & $241(69.1)$ & $14(82.4)$ \\
\hline Diabetes & $50(13.7)$ & $46(13.2)$ & $4(23.5)$ \\
\hline COPD & $17(4.6)$ & $16(4.6)$ & $1(5.9)$ \\
\hline Kidney disease & $13(3.6)$ & $12(3.4)$ & $1(5.9)$ \\
\hline Sleep apnoea & $17(4.6)$ & $16(4.6)$ & $1(5.9)$ \\
\hline \multicolumn{4}{|l|}{ Smoking (pack years) } \\
\hline None & $118(32.2)$ & $111(31.8)$ & $7(41.2)$ \\
\hline $1-20$ & $76(20.8)$ & 72 (20.6) & $4(23.5)$ \\
\hline$>20$ & $172(47)$ & $166(47.6)$ & $6(35.3)$ \\
\hline Excessive alcohol intake & $44(12)$ & $42(12)$ & $2(11.8)$ \\
\hline Married/partner & $269(73.5)$ & $255(73.1)$ & $14(82.4)$ \\
\hline Nursing home & $1(0.3)$ & $1(0.3)$ & 0 \\
\hline No acute infarct on imaging & $163(44.5)$ & $158(45.3)$ & $5(29.4)$ \\
\hline Acute lacunar infarct & $87(23.8)$ & $87(24.9)$ & 0 \\
\hline Acute non-lacunar infarct & $91(24.9)$ & $81(23.2)$ & $10(58.8)$ \\
\hline Ultrasound or angiography conducted & $322(88)$ & $308(88.3)$ & $14(82.4)$ \\
\hline Occlusion or stenosis* & 70 (19.1) & $65(18.6)$ & $5(29.4)$ \\
\hline Prior cECG & $148(40.4)$ & $140(40.1)$ & $8(47.1)$ \\
\hline Chronic infarction & $180(49.2)$ & $174(49.9)$ & $6(35.3)$ \\
\hline \multicolumn{4}{|l|}{ TOAST classification } \\
\hline Small artery occlusion & $169(46.2)$ & $165(47.3)$ & $4(23.5)$ \\
\hline Cardioembolism & 0 & 0 & 0 \\
\hline Large-artery atherosclerosis & $43(11.7)$ & $39(11.2)$ & $4(23.5)$ \\
\hline Undetermined & $149(40.7)$ & $140(40.1)$ & $9(52.9)$ \\
\hline Othert & $5(1.4)$ & $5(1.4)$ & 0 \\
\hline Pulse & $70(62-78)$ & $70(62-78)$ & $68(61-75)$ \\
\hline Systolic BP & $141.5(131-153)$ & $142(131-153)$ & $137(132-153)$ \\
\hline Diastolic BP & $83(77-92)$ & $84(77-92)$ & $82(70-87)$ \\
\hline $\mathrm{BMI}\left(\mathrm{kg} / \mathrm{m}^{2}\right)$ & $26.9(24.2-29.9)$ & $26.8(24.1-30)$ & $28.2(26.7-29)$ \\
\hline LDL-C (mmol/L) & $1.8(1.4-2.3)$ & $1.8(1.4-2.3)$ & $2(1.3-2.2)$ \\
\hline Dyslipidaemia (LDL >1.8 $\mathrm{mmol} / \mathrm{L})$ & $176(48.1)$ & $165(47.3)$ & $11(64.7)$ \\
\hline Creatinine $(\mu \mathrm{mol} / \mathrm{L})$ & $79(67-92)$ & $78(66-92)$ & $85(75-96)$ \\
\hline $\mathrm{HbA1C}(\mathrm{mmol} / \mathrm{mol})$ & $38(36-41)$ & $38(36-41)$ & $40(37-42)$ \\
\hline NT-proBNP (pg/mL) & $105.3(51.2-211.4)$ & $101.5(48.2-208)$ & $191.1(92.2-339.1)$ \\
\hline NT-proBNP $\leq 200 \mathrm{pg} / \mathrm{mL}$ & $267(73)$ & $258(73.9)$ & $9(52.9)$ \\
\hline NT-proBNP 199-399 pg/mL & $39(10.7)$ & $36(10.3)$ & $3(17.6)$ \\
\hline NT-proBNP $\geq 400 \mathrm{pg} / \mathrm{mL}$ & $60(16.4)$ & $55(15.8)$ & $5(29.4)$ \\
\hline
\end{tabular}

All categorical data are expressed as $n(\%)$.

The variables years since last stroke, $\mathrm{CHA}_{2} \mathrm{DS}_{2}$-VASC score, pulse, BP, BMI, LDL-C, creatinine, HbA1C and NT-proBNP are expressed with median (Q1-Q3).

$* 50 \%$ occlusion or stenosis.

†Vascular dissection and in one patient vasculitis was suspected.

$\mathrm{BMI}$, body mass index; $\mathrm{BP}$, blood pressure; $\mathrm{CECG}$, continuous ECG; $\mathrm{CHA}_{2} \mathrm{DS}_{2}$-VASc, Congestive heart failure, Hypertension, Age, Diabetes, previous Stroke/transient ischemic attack,

Vascular disease, Sex category; COPD, chronic obstructive pulmonary disorder; HbA1C, haemoglobin A1C; LDL-C, low-density lipoprotein cholesterol; NT-ProBNP, N-terminal Pro-

Brain Natriuretic Peptide (NT-proBNP); TIA, transient ischaemic attack; TOAST, Trial of Org 10172 in Acute Stroke Treatment. 
A



Figure 2 Distribution of the total burden of AF during 7-day Holter monitoring in participants with ischaemic stroke more than 1 year before inclusion. Displayed with absolute values $(A)$ and proportion of readable Holter ECG (B). AF, atrial fibrillation.

of interpretable Holter data was 6.9 days (Q1-Q3 6.8-7.0). Online supplementary appendix table S4 depicts the distribution of completed Holter durations. Of the participants, $>93 \%$ completed more than 6 days of Holter monitoring. Pulse palpation revealed irregular pulse in 122 of 366 participants $(33 \%$ (95\% CI 28.5 to 38.4$)$ ). The 12-lead ECG showed AF in 3 of 366 participants $(0.82 \%$ (95\% CI 0.17 to 2.4$)$ ), and after 7 -day Holter monitoring AF was detected in 17 of 366 participants (4.6\% (95\% CI 2.7 to 7.3$)$ ). The number needed to screen with 7 -day Holter monitoring was 22 (14-37). Since no AF detected by 12-lead ECG was missed during 7-day Holter monitoring, the proportion of $\mathrm{AF}$ uncovered by the complete evaluation was equivalent to the proportion achieved by Holter monitoring. Figure 2 depicts the distributions of the time in AF during Holter monitoring. Absolute values and proportions are displayed in online supplementary appendix table S5. Most participants had AF for less than $1 \%$ of the time (10 of 17 AF cases).

\section{Pulse palpation and 12-lead ECG}

Table 2 is a $2 \times 2$ contingency table outlining the distribution of outcomes between evaluation methods. Using 7-day Holter monitoring as a reference standard, pulse palpation missed 9 of 17 participants with AF. The 12-lead ECG missed 14 of 17 participants with AF. The sensitivity of pulse palpation was $47 \%$ (95\% CI $23 \%$ to $72 \%)$ and the sensitivity of 12-lead ECG was $18 \%$ (95\% CI $4 \%$ to $43 \%$ ) (table 3 ).

\begin{tabular}{|c|c|c|}
\hline \multirow[b]{2}{*}{ Pulse palpation } & \multicolumn{2}{|c|}{ 7-day Holter monitoring } \\
\hline & $+\mathrm{AF}$ & $-\mathrm{AF}$ \\
\hline Pulse irregular & 8 & 114 \\
\hline \multirow[t]{2}{*}{ Pulse regular } & 9 & 235 \\
\hline & \multicolumn{2}{|c|}{ 7-day Holter monitoring } \\
\hline 12-lead ECG & $+\mathrm{AF}$ & $-\mathrm{AF}$ \\
\hline $\mathrm{AF}$ & 3 & 0 \\
\hline \multirow[t]{2}{*}{ Sinus rhythm } & 14 & 349 \\
\hline & \multicolumn{2}{|c|}{ 12-lead ECG } \\
\hline Pulse palpation & $+\mathrm{AF}$ & $-\mathrm{AF}$ \\
\hline Pulse irregular & 2 & 120 \\
\hline Pulse regular & 1 & 243 \\
\hline
\end{tabular}

$\mathrm{AF}$, atrial fibrillation.
Table 3 Proportions of detected AF and sensitivity, specificity, PPV and NPV for detecting AF by pulse palpation and 12-lead ECG holding 7-day Holter monitoring as a reference standard

\begin{tabular}{|c|c|c|c|c|}
\hline & \multicolumn{4}{|c|}{ Accuracy in $\%(95 \% \mathrm{Cl})$} \\
\hline & Sensitivity & Specificity & PPV & NPV \\
\hline Pulse irregular & 47 (23 to 72 ) & 67 (62 to 72$)$ & 7 (3 to 13$)$ & 96 (93 to 98) \\
\hline 12-lead ECG & 18 (4 to 43$)$ & $100(99 \text { to } 100)^{*}$ & 100 (30 to 100$)$ & 96 (94 to 98) \\
\hline $\begin{array}{l}\text { 7-day Holter } \\
\text { monitoring }\end{array}$ & \multicolumn{4}{|c|}{ Reference standard } \\
\hline
\end{tabular}

\section{Clinical factors associated with AF}

Increasing age was associated with increased odds of $\mathrm{AF}$ $(\mathrm{OR}=1.1,95 \% \mathrm{CI}(1.02$ to 1.15$))$. There was no significant increase in the odds of AF for participants with high levels of NT-proBNP $(\geq 400 \mathrm{pg} / \mathrm{mL})$ compared with lower levels $(\leq 200 \mathrm{pg} / \mathrm{mL})$, and neither in the univariate analysis nor when adjusting for age (OR 2.4 (95\% CI 0.5 to 8.4 ) and $1.6(95 \%$ CI 0.3 to 6.0$)$ ). There was no significant difference in the time passed since stroke between participants with and without AF (online supplementary appendix table S2).

None of the participants with AF had a secondary precipitant (infection, anaemia, thyroid disease, recent surgery). At baseline $58 \%$ of participants $(n=222)$ reported cardiopulmonary symptoms experienced at least within the last 3 months prior to inclusion. Cardiopulmonary symptoms were not associated with increased odds of AF (online supplementary appendix table S6).

In a sensitivity analysis we added participants excluded due to transient ischaemic attack or stroke over 10 years prior to our population. The proportion of participants with AF in this population was $4.4 \%$ (95\% CI 2.6 to 7.0$)$. The remaining analyses remained robust.

\section{DISCUSSION}

Screening patients in a primary care setting over 1 year poststroke using 7-day Holter monitoring uncovered AF in $4.6 \%$ of participants without known AF. Compared with 7-day Holter monitoring, more than half of AF diagnoses were undetectable with pulse palpation and more than $82.3 \%$ were missed by 12-lead ECG. High versus low levels of NT-proBNP were not significantly associated with AF. Without the use of cECG, in addition to opportunistic pulse palpation and routine 12-lead ECG, a considerable number of patients with AF may be underdiagnosed during routine poststroke AF screening in the primary sector.

\section{Previous studies}

cECG and AF yield

Previous studies of poststroke AF detection focused on the period immediately after stroke. A meta-analysis including 31 studies estimated an expected AF detection rate by 7-day monitoring of $8.9 \% .^{15}$ Similarly, 10-day Holter monitoring identified AF in $9 \%$ in the Find-AF(RANDOMISED) trial. ${ }^{7}$ The comparatively lower detection rate found in our study may be explained by a time-related dilution of participants with AF for several reasons. First is the exclusion of participants diagnosed with AF before inclusion. Second is the attrition of participants with undiagnosed $\mathrm{AF}$ due to the high risk of a recurrent stroke and death. $^{216}$ Third, AF is associated with more severe strokes ${ }^{17}$ and therefore such patients more often reside at a nursing home. Due to practical issues, only patients living in their own home were 
approached for participation in this study. Further, study participation required the ability to understand and cooperate during the study. Consequently, the results of this study may reflect a subset of patients who had a stroke with lower comorbid burden and less severe strokes.

\section{Pulse palpation and 12-lead ECG versus CECG}

Irregular pulse felt during pulse palpation could be attributed to non-AF arrhythmias such as frequent atrial or ventricular ectopy or sinus arrhythmia. The sensitivity and specificity of pulse palpation were higher in previous studies (92\% and $82 \%)$ than in the present study (47\% and 68.2\%). ${ }^{13}$ This could be explained by the use of 12-lead ECG as a reference standard instead of continuous monitoring.

The AF yield achieved by 12-lead ECG in this study $(0.82 \%$ (95\% CI 0.17 to 2.4$)$ ) was in line with previous studies. A metaanalysis and a large Danish observational multicentre study investigated primary care screening of participants 65 years or older, whereof 5\%-7\% previously had a stroke. ${ }^{18} 19$ AF was uncovered in $1 \%-1.4 \%$ of the screened. ${ }^{18} 19$ The study population was younger in our study, but having a previous stroke is a likely marker of increased risk of $\mathrm{AF}^{20}$ Our study showed a substantially increased AF detection with cECG compared with 12-lead ECG. Studies involving primary care screening of nonstroke patients have shown similar results. The STROKESTOP study screened Swedish residents aged 75-76 years old with repeated thumb placement on a handheld ECG for 2 weeks. ${ }^{21}$ AF was uncovered in 3\%, a fourfold increase compared with baseline 12-lead ECG. Further, the addition of cECG to 12-lead ECG after acute stroke has proved beneficial in various studies; however, studies differ in terms of age, stroke subtype and cECG monitoring length and methods.

\section{NT-proBNP and AF}

Opposed to findings in previous studies, our study showed that increased NT-proBNP levels did not seem useful as a selection parameter for extended AF screening. ${ }^{22}$ The optimal cut-off value differed across these studies, but even a comparison of assumed low and high risk values as previously suggested ${ }^{23}$ showed no significant association to AF in the present study. A lack of power for this secondary endpoint may explain our finding.

\section{Relation between $\mathrm{AF}$ and prior stroke}

The time passed since ischaemic stroke was several years for most participants, why all AF cases may not be causally linked to the index stroke. AF is associated with large vessel embolic occlusions ${ }^{24}$ and a causal link is supported by the presence of an acute non-lacunar infarct on cerebral imaging in all participants diagnosed with AF where an acute infarct was documented. Further, a low AF burden and a considerable proportion of participants with cryptogenic stroke (40.7\%) might suggest the presence of an 'embolic stroke of undetermined source', such as AF, which is described by Hart et al. ${ }^{25} \mathrm{AF}$ could have been coexisting because risk factors of AF and stroke overlap. ${ }^{26}{ }^{27}$ Further, Kamel et al ${ }^{28}$ described an 'atrial substrate model' in which atrial myopathy may create an atrial substrate with independent thromboembolic qualities. This means the presence of an atrial substrate may cause stroke without the presence of AF. AF may in turn be a 'lagging marker of thrombogenic atrial abnormalities'. ${ }^{28}$ Additionally, the low AF burden detected in our study could be indicative of a relatively recently developed AF. Although speculative, the atrial substrate model therefore seems to explain both why all participants with AF had non-lacunar strokes and it accounts for the long time passed between index stroke and AF. ${ }^{28}$

\section{Clinical implications}

Following current guidelines, ischaemic strokes yield two points on the $\mathrm{CHA}_{2} \mathrm{DS}_{2}$-VASc scale, and a shift from antiplatelet to OAC treatment is recommended independently of causal connection. ${ }^{8}$ However, the necessary AF duration and burden associated with a prognostic benefit of OAC are unknown, and more patients with low burden AF will be caught using continuous monitoring compared with using 12-lead ECG. A low AF burden has been associated with a lower stroke recurrence risk than high burden $\mathrm{AF}^{29}$ Consequently, one may question the prognostic benefits of more aggressive systematic screening during poststroke primary care follow-up. On the other side, AF is considered a progressive disease, deteriorating with age and comorbid burden. ${ }^{8}$ Further, cECG is still a snap shot of a relatively short time window, leaving us unable to determine the true AF burden over a longer time period. These factors and a high stroke recurrence risk and mortality in untreated $\mathrm{AF}^{216}$ support a need to improve $\mathrm{AF}$ detection even long after stroke.

There is currently no evidence that intensive AF screening is independently associated with a reduction in stroke recurrence and mortality. Ongoing studies including patients who had acute stroke aim to clarify this question. ${ }^{30}$

\section{Limitations and strengths}

A high proportion of participants complained of cardiopulmonary symptoms, but these were not associated with increased odds of AF. There was no control group, which is why we cannot preclude that the participants would have been diagnosed later without our interference. There is therefore a

\section{Key messages}

\section{What is already known on this subject?}

- Uncovering atrial fibrillation after stroke is imperative because a shift to oral anticoagulant treatment effectively reduces stroke recurrence and mortality.

- Atrial fibrillation often remains undiagnosed, but use of continuous ECG after acute stroke is superior to single-point ECG evaluations.

- There are no guidelines on atrial fibrillation detection long after stroke and no studies have investigated the diagnostic value of using continuous ECG for poststroke screening in primary care.

What might this study add?

- Using 7-day Holter monitoring in participants with stroke over 1 year before inclusion, in a primary care setting, revealed new-onset atrial fibrillation in a relevant proportion of participants.

- 7-day Holter monitoring substantially improved the detection rate compared with baseline pulse palpation and 12-lead ECG.

How might this impact on clinical practice?

- The results suggest that continuous ECG methods should be considered for poststroke atrial fibrillation screening even long after stroke.

- They further question the lack of guidelines for atrial fibrillation screening during poststroke primary care. 
risk of selection bias, affecting external validity. A subset of participants had a chronic infarct on imaging without certain clinical evidence of recent stroke. This limits the possibility of assessing time lag since last stroke, which may have been underestimated. Further, the risk of AF may be different in these participants compared with participants with clinical infarcts, potentially diluting our results. Our study is strengthened by a large sample size and a low dropout rate. Further, the heterogeneity of stroke subtypes mimics conditions in real life, thereby increasing generalisability.

\section{CONCLUSIONS}

We showed that 7-day Holter monitoring for poststroke AF detection in a primary care setting uncovered a relevant number of AF cases and substantially improved the detection rate compared with pulse palpation and 12-lead ECG. Therefore, cECG seems relevant not only for AF detection in the acute phase after ischaemic stroke, but also as an additional tool for AF screening in GP clinics long after stroke. Further studies investigating primary sector AF screening long after stroke would be needed to clarify the prognostic impact, the benefit and feasibility of different cECG devices, and to identify which markers of AF could be used as a selection tool for extended screening.

Acknowledgements We thank all those who participated in this study or who expressed interest in participating. We also thank the research assistants and the staff at the Department of Neurology Zealand University Hospital and all staff at both GP clinics for the collaboration. We in particular thank Hanne Bjørnum (RN), Karen Larsen (RN), Thomas Andersen (GP) and research assistant Julie Kasten Rønne for all their important help in the data collection process. Thomas Andersen and the Department of Neurology provided resources and facilities for participant recruitment and analysis. At Borup GP clinic Hanne Bjørnum recruited the participants, managed the logistics, carried out the data collection and supervised the training of other data collectors. Karen Larsen and Julie Kasten Rønne collected the data at Havdrup GP clinic NVC, respectively.

Contributors JCT, SLL and TW formulated the idea. LFL drafted the protocol, designed the study, recruited the patients, collected the data, analysed the data and drafted the manuscript. TW provided critical review of the protocol. SLL provided resources and facilities for participant recruitment and analysis. LFL analysed the ECG data, and AMA, MLH and BB provided critical reviews of ECG events. JCT was consulted for clinical cardiac questions during the study. TW and LFL acquired financial support. TW and MLH supervised the research activities. All authors critically revised and approved the final version of the manuscript.

Funding The study was supported by Bayer, Boehringer Ingelheim, the Department of Neurology, Zealand University Hospital, 'Region Sjællands Ordinære pulje', 'Grosserer L.F. Foghts Fond', 'A.P. Møller og Hustru Chastine Mc-Kinney Møllers Fond til almene Formaal-Fonden til Lægevidenskabens Fremme' and 'Hans og Nora Buchards Fond'. The sponsors had no role in the conception, drafting the protocol, conducting the analyses or interpreting the results.

Competing interests LFL has competing interests as stated under the Funding section and personal fees from Bayer, outside the submitted work. TW reports personal fees from Boehringer Ingelheim and Bayer, outside the submitted work. MLH, JCT, SLL, BB and AMA have no disclosures.

Patient and public involvement Patients and/or the public were not involved in the design, or conduct, or reporting, or dissemination plans of this research.

Patient consent for publication Not required.

Ethics approval The study was conducted in accordance with the Declaration of Helsinki. The Region Zealand Committee of Health Research Ethics and the Danish Data Protection Agency approved the protocol.

Provenance and peer review Not commissioned; externally peer reviewed.

Data availability statement Anonymised data are available upon reasonable request.

Open access This is an open access article distributed in accordance with the Creative Commons Attribution Non Commercial (CC BY-NC 4.0) license, which permits others to distribute, remix, adapt, build upon this work non-commercially, and license their derivative works on different terms, provided the original work is properly cited, appropriate credit is given, any changes made indicated, and the use is non-commercial. See: http://creativecommons.org/licenses/by-nc/4.0/.

\section{ORCID iD}

Louise Feldborg Lyckhage http://orcid.org/0000-0003-4866-1169

\section{REFERENCES}

1 Benjamin EJ, Muntner P, Alonso A, et al. Heart disease and stroke Statistics-2019 update: a report from the American heart association. Circulation 2019;139:e56-28.

2 Wolf PA, Abbott RD, Kannel WB. Atrial fibrillation as an independent risk factor for stroke: the Framingham study. Stroke 1991;22:983-8.

3 Hart RG, Pearce LA, Aguilar MI. Meta-Analysis: antithrombotic therapy to prevent stroke in patients who have nonvalvular atrial fibrillation. Ann Intern Med 2007; 146:857-67.

4 Healey JS, Connolly SJ, Gold MR, et al. Subclinical atrial fibrillation and the risk of stroke. N Engl J Med 2012;366:120-9.

5 Sposato LA, Cipriano LE, Saposnik G, et al. Diagnosis of atrial fibrillation after stroke and transient ischaemic attack: a systematic review and meta-analysis. Lancet Neurol 2015;14:377-87

6 Brachmann J, Morillo CA, Sanna T, et al. Uncovering atrial fibrillation beyond short-term monitoring in cryptogenic stroke patients: three-year results from the cryptogenic stroke and underlying atrial fibrillation trial. Circ Arrhythm Electrophysiol 2016;9:e003333.

7 Wachter R, Gröschel K, Gelbrich G, et al. Holter-electrocardiogram-monitoring in patients with acute ischaemic stroke (Find- $\left.\mathrm{AF}_{\text {RANDOMISED }}\right)$ : an open-label controlled trial. Lancet Neurol 2017:16:282-90.

8 Kirchhof P, Benussi S, Kotecha D, et al. 2016 ESC Guidelines for the management of atrial fibrillation developed in collaboration with EACTS: The Task Force for the management of atrial fibrillation of the European Society of Cardiology (ESC) Developed with the special contribution of the European Heart Rhythm Association (EHRA) of the ESCEndorsed by the European Stroke Organisation (ESO). Eur Heart $J$ 2016:ehw210.

9 Sacco RL, Kasner SE, Broderick JP, et al. An updated definition of stroke for the 21st century: a statement for healthcare professionals from the American heart Association/American stroke association. Stroke 2013;44:2064-89.

10 Smith EE, Saposnik G, Biessels GJ, et al. Prevention of stroke in patients with silent cerebrovascular disease: a scientific statement for healthcare professionals from the American heart Association/American stroke association. Stroke 2017:48:e44-71.

11 Adams HP, Bendixen BH, Kappelle LJ, et al. Classification of subtype of acute ischemic stroke. definitions for use in a multicenter clinical trial. TOAST. trial of ORG 10172 in acute stroke treatment. Stroke 1993;24:35-41.

12 Morgan S, Mant D. Randomised trial of two approaches to screening for atrial fibrillation in UK general practice. Br J Gen Pract 2002;52:377-80.

13 Taggar JS, Coleman T, Lewis $\mathrm{S}$, et al. Accuracy of methods for detecting an irregular pulse and suspected atrial fibrillation: a systematic review and meta-analysis. Eur J Prev Cardiol 2016:23:1330-8

14 Haeberlin A, Roten L, Schilling M, et al. Software-based detection of atrial fibrillation in long-term ECGs. Heart Rhythm 2014;11:933-8.

15 Dussault C, Toeg H, Nathan M, et al. Electrocardiographic monitoring for detecting atrial fibrillation after ischemic stroke or transient ischemic attack: systematic review and meta-analysis. Circ Arrhythm Electrophysiol 2015;8:263-9.

16 Benjamin EJ, Wolf PA, D'Agostino RB, et al. Impact of atrial fibrillation on the risk of death: the Framingham heart study. Circulation 1998;98:946-52.

17 Lin HJ, Wolf PA, Kelly-Hayes M, et al. Stroke severity in atrial fibrillation. The Framingham study. Stroke 1996;27:1760-4.

18 Lowres N, Neubeck L, Redfern J, et al. Screening to identify unknown atrial fibrillation. A systematic review. Thromb Haemost 2013;110:213-22.

19 Hald J, Poulsen PB, Qvist I, et al. Opportunistic screening for atrial fibrillation in a real-life setting in general practice in Denmark-The atrial fibrillation found on routine detection (afford) non-interventional study. PLoS One 2017:12:e0188086

20 Fauchier L, Clementy N, Pelade C, et al. Patients with ischemic stroke and incident atrial fibrillation: a nationwide cohort study. Stroke 2015:46:2432-7.

21 Svennberg E, Engdahl J, Al-Khalili F, et al. Mass screening for untreated atrial fibrillation: the STROKESTOP study. Circulation 2015;131:2176-84.

22 Sepehri Shamloo A, Bollmann A, Dagres N, et al. Natriuretic peptides: biomarkers for atrial fibrillation management. Clin Res Cardiol 2020;33:1-3.

23 Haeusler KG, Gröschel K, Köhrmann M, et al. Expert opinion paper on atrial fibrillation detection after ischemic stroke. Clin Res Cardiol 2018;107:871-80.

24 Bang OY, Ovbiagele B, Kim JS. Evaluation of cryptogenic stroke with advanced diagnostic techniques. Stroke 2014;45:1186-94.

25 Hart RG, Diener H-C, Coutts SB, et al. Embolic strokes of undetermined source: the case for a new clinical construct. Lancet Neurol 2014;13:429-38. 
26 Andrade J, Khairy P, Dobrev D, et al. The clinical profile and pathophysiology of atrial fibrillation: relationships among clinical features, epidemiology, and mechanisms. Circ Res 2014;114:1453-68.

27 Guzik A, Bushnell C. Stroke epidemiology and risk factor management. Continuum 2017;23:15-39.

28 Kamel H, Okin PM, Elkind MSV, et al. Atrial fibrillation and mechanisms of stroke: time for a new model. Stroke 2016;47:895-900.
29 Go AS, Reynolds K, Yang J, et al. Association of burden of atrial fibrillation with risk of ischemic stroke in adults with paroxysmal atrial fibrillation: the KP-RHYTHM study. JAMA Cardiol 2018;3:601-8.

30 Haeusler KG, Kirchhof P, Heuschmann PU, et al. Impact of standardized monitoring for detection of atrial fibrillation in ischemic stroke (MonDAFIS): rationale and design of a prospective randomized multicenter study. Am Heart J 2016;172:19-25. 